\title{
Neuromuscular transmission in human single motor units
}

\author{
H.A.K A D R I E A N W F. B R O W N \\ From University Hospital, London, Ontario, Canada
}

SUMMARY A method, multiple point stimulation, has been reported to isolate 5-20 single hypothenar or thenar motor units for investigation. This method is attractive for testing neuromuscular transmission because the required stimulus intensities are much less than for supramaximal nerve stimulation, and movement artefact is less of a problem. In this investigation of controls, the changes in hypothenar and thenar motor unit surface voltage and latency of single muscle fibre action potentials belonging to the motor unit have been measured in response to trains of stimuli delivered to the motor nerve. In healthy motor units, increases in the surface peak-to-peak voltage and corresponding reductions in the peak-to-peak duration occurred, the changes being maximum at the shorter stimulus intervals and accompanied by increased synchronisation of motor unit muscle fibre action potential discharges. The investigations of the responses for healthy single motor units to repetitive stimulation provide the basis for investigations of neuromuscular transmission at the level of the motor unit in diseases of neuromuscular transmission.

The most common electrophysiological test of neuromuscular transmission in humans is to measure the changes in force or voltage output from muscle(s) evoked by trains of supramaximal stimuli delivered to the motor nerve (Slomic et al., 1968; Ozdemir and Young, 1971, 1976; Desmedt, 1973; Desmedt and Borenstein, 1976). Unfortunately, this type of test can be uncomfortable and distorted by movement artefact, and muscles like the hand muscles, technically the best to test, are not clinically the most commonly or severely involved in myasthenia gravis (Simpson, 1974). At the expense of more time and patient discomfort, the diagnostic usefulness of supramaximal stimulation tests can be increased by testing a number of muscles including proximal limb and even facial muscles (Ozdemir and Young, 1971, 1976; Desmedt and Borenstein, 1970, 1976; Borenstein and Desmedt, 1973; Krarup, 1977a, b). The limitations of supramaximal stimulation led us to question if neuromuscular transmission could be tested practically in single motor units (MU). Such a method would have the probable advan-

Address for reprint requests: Dr W. F. Brown, University Hospital, 339 Windermere Road, PO Box 5339, Postal Stn A, London, Ontario, Canada N6A 5A5.

Accepted 26 September 1977 tages of less movement artefact, and lower, and therefore more comfortable, stimulus intensities, and the theoretical advantage of testing the basic unit of motor control, the MU.

Important landmarks in the testing of the physiological properties of human single MUs and axons in health and disease include the thorough investigations by Bergmans (1970) and Desmedt and Borenstein (1970). Indeed, the fact that posttetanic exhaustion and pathological decrements in response to trains of presynaptic stimuli were not present in all myasthenic MUs suggested to Desmedt (1973) that certain types of MUs may be more involved than others. Desmedt and Borenstein (1970), however, pointed to the difficulties of isolating even one MU per test subject. As a consequence, testing neuromuscular transmission at the level of the individual MU did not look like a practical test for most laboratories.

From this laboratory, a method has been reported, using graded threshold electrical stimulation to isolate four to 20 or more distinct MUs per test nerve (Kadrie et al., 1976). In preference to the needle stimulating electrodes that complicated the technical procedure used by Desmedt and Borenstein (1970), surface stimulation of nerve has been preferred by us as the better 
method of stimulation; the result being a much larger number of MUs isolated per test session. It is our intention, in this communication, to establish the normal observations for tests of neuromuscular transmission in single MUs. In later papers, we will report on our observations in disorders of neuromuscular transmission including myasthenia gravis and motor neurone disease.

Unfortunately, the force generated by individual MUs isolated by stimulation of the motor nerve could not be measured properly, nor could the conduction velocities of single axons be measured directly because MUs were isolated by stimulation at single points that varied from $\mathrm{MU}$ to $\mathrm{MU}$ in distance from the motor point. Therefore, to establish the probable functional type and size of MU, it was necessary to rely on the surface voltage, a parameter known to be approximately related to the MU force (Milner-Brown and Stein, 1975) and the latency (or conduction velocity) of the MUs adjusted to a standard distance from the motor point (Kadrie et al., 1976). The latter represented an important second indicator of the probable functional type and size of the MU isolated by nerve stimulation because of the established relationship between MU tension and conduction velocity (Freund et al., 1975).

An attempt was made also to combine a look at the overall action of the MU, reflected in the surface voltage and the component single neuromuscular junctions, the latter recorded by means of the single fibre (SF-EMG) electrode. Unfortunately, single muscle fibre action potentials from two or more fibres were recorded from only three MUs in parallel to the surface voltage measurement. Observations in the three MUs were consistent, however, in pointing to increased synchronisation as the probable most important mechanism behind pseudofacilitation.

\section{Subjects and methods}

A total of 12 ulnar and two median nerves were tested in 12 healthy subjects (age range 14-36 years). The median nerves were included in order to establish if the range in $\mathrm{MU} \mathrm{p}-\mathrm{pV}$ and relationship of MU adjusted latency to surface peak-peak voltage (p-pV) were the same for median as for ulnar control nerves.

\section{ELECTROPHYSIOLOGICAL METHODS}

The method for multiple point stimulation (Kadrie et al., 1976) has been modified for this investigation. In the original method, signal averaging of the "all" or "nothing" MU responses, and subtraction of the "nothing" from the "all" MU potentials by means of the digital computer was used to measure the $\mathrm{p}-\mathrm{pV}$, area and latencies of the MUs. For this investigation, the computer was not necessary because most of the MUs tested were the first recruited; hence subtraction was not required. Moreover, in the earlier investigations, the changes in the surface $p-p V$ for the most part paralleled changes in area, making the latter measurement unnecessary.

Signal averaging cannot be used to measure the parameters of MUs with abnormal neuromuscular transmission because of changes in the MU evoked by presynaptic stimuli even at stimulus intervals of less than one second. Moreover, experience has taught us that measurement of MU latencies by "eye" is more accurate, particularly for MUs that have a low signal to noise ratioprecisely the situations in which the computer occasionally made errors in the recognition of the point of initial deflection. For the purpose of this investigation, only MUs that had a clearly defined initial deflection were included in the MU sample for adjustment of the latency to the standard $200 \mathrm{~mm}$.

An intramuscular electrode was used to help identify the point of initial deflection, exclude the possible contribution of more than one MU to the surface voltage, and investigate the changes at one or more neuromuscular junctions belonging to the presynaptically stimulated MU. For the latter, a TECA single fibre EMG (SF-EMG) electrode was used, the electrode being located close to the endplate zone of the hypothenar or thenar muscle complexes. In the SF-EMG recordings, measurements were made of the variation in the distal latency to single spikes from the stimulus artefact and changes in the interspike intervals between single muscle fibres belonging to the same MU.

Especially for the measurement of changes in the parameters of the maximum compound potential, it was important to minimise movement artefact by firmly binding the hand and arm, including the first digit in the case of the median-thenar muscle testing, to a test board.

\section{METHOD OF STIMULATION}

A DISA constant current type 15E07 stimulator was used, the stimulus duration being $0.05-0.1$ milliseconds (ms). This stimulator facilitated comparisons of the threshold of single MUs obtained at different points with the intensities required to stimulate the motor nerve in a supramaximal manner. The ulnar and median nerves were stimulated by means of a DISA surface $13 \mathrm{~K} 62$ bipolar electrode held by hand. 
Attempts to obtain large numbers of MUs were frustrated and delayed by trying to hold the stimulating electrode in position mechanically by means of straps or tape. Holding the electrode by hand was a better method that made it possible to stimulate, in isolation, single MUs for as long as 30 minutes. It was frequently possible to obtain the identical MU at the same location half to one hour later. Minor changes in position or pressure were often all that was required to make the difference between success and failure in isolating single MUs, particularly in the upper arm. Once learned, this method made it possible to obtain at least four and frequently 20 or more single MUs from the ulnar or median nerve in less than an hour. In our hands, needle stimulating electrodes (Desmedt and Borenstein, 1970) made it harder to isolate single MUs and certainly added to the discomfort of the procedure.

\section{RECORDING AMPLIFIERS}

The intramuscular and surface voltage changes were recorded using DISA type 15C01 EMG amplifiers that have a peak-peak noise at $5 \mathrm{k}$ amplification of $0.003-0.004 \mathrm{mV}$. All the data were recorded on magnetic tape and later photographed for careful measurement and analysis.

\section{PARAMETERS MEASURED}

Maximum compound potential $=$ maximum conduction velocity

For the maximum compound potential (MCP) the p-pV, peak-to-peak duration (p-pD) and distal latency from stimulus artefact to initial deflection of the motor unit potential were measured. The maximum conduction velocities (MCV) were calculated for standard distances of $400 \mathrm{~mm}$ from the motor point by:

$$
\begin{aligned}
& \mathrm{MCV} \text { in } \mathrm{m} / \mathrm{s}= \\
& \frac{400}{\mathrm{MCP} \text { latency projected to } 400 \mathrm{~mm}-\mathrm{RL}}
\end{aligned}
$$

\section{Residual latency}

To obtain the residual latency (RL) the motor nerve was stimulated at three or more points distributed over the length of the nerve from the proximal upper arm to the wrist; the latency being plotted against distance from the motor point to obtain the RL (Fig. 1). The RL was later used to calculate the adjusted latencies of the single MUs. In all controls, the correlation coefficients for the three to four stimulation points used to obtain the residual latency were high $(>0.99)$.

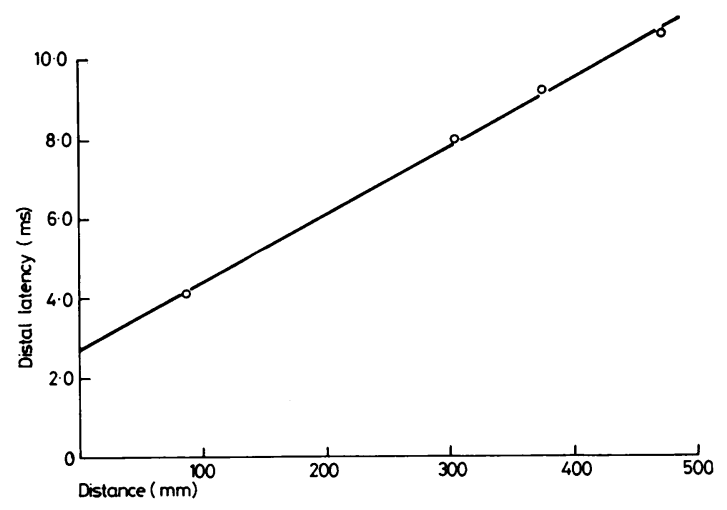

Fig. 1 In a representative healthy subject a plot of the latencies in milliseconds from the points of stimulation on the ulnar nerve to the initial deflection of the maximum compound potential. Residual latency was measured by projection of the line joining the four stimulation points to the $Y$ axis. The latency at the intersect is the residual latency $(R L)$

\section{Single $M U$ parameters}

For each MU, the distal latency, surface p-pV, and current intensity to obtain a firing index more than $50 \%$ were measured and recorded. At most points only one MU could be obtained that fired $100 \%$ of the time in response to the nerve stimulus in the absence of evidence of overlap in the firing levels with other MUs (Brown and MilnerBrown, 1976). This condition is obviously required for testing neuromuscular transmission using trains of stimuli. At a minority of points a second, or sometimes a third, MU could be obtained by increments in the stimulus intensity, the critical firing level of each MU in turn being clearly separate, so that the size and latency of the MU isolated at higher stimulus intensities could be measured. Motor units are recruited in order from small to larger p-pV by increments in the stimulus intensity (Kadrie et al., 1976). To include more medium $(0.05-0.149 \mathrm{mV} \mathrm{p}-\mathrm{pV})$ and large $(>0.150 \mathrm{mV} \mathrm{p}-\mathrm{pV}) \mathrm{MUs}$ an effort was made to avoid the more readily obtainable small MUs in order to find the occasional larger MU firing at threshold in preference to recruitment second or third in order (Fig. 2).

\section{Criteria for a single unit}

The criteria for a single unit response (Fig. 3) were: (1) all or nothing response in the surface and intramuscular records evoked by stimulus shocks at threshold for the MU; (2) no evidence of overlap in the firing levels of two or more MUs evoked by trains of 25-100 stimuli (Brown and 


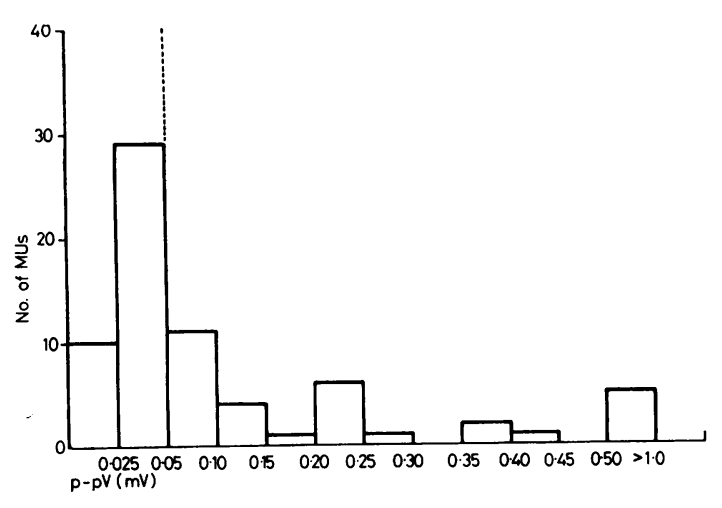

Fig. 2 Histogram of control hypothenar motor units first excited. A deliberate attempt was made to find points of stimulation where medium and large MUs could be excited first. As a consequence, $a$ higher proportion of larger MUs are included in this $M U$ population compared to that where MUs obtained from all points of stimulation were included (Kadrie et al., 1976). Broken lines at $0.05 \mathrm{mV}$ point to the fact that the first two divisions on the abscissa are $0.025 \mathrm{mV}$, while subsequent divisions are $0.05 \mathrm{mV}$.

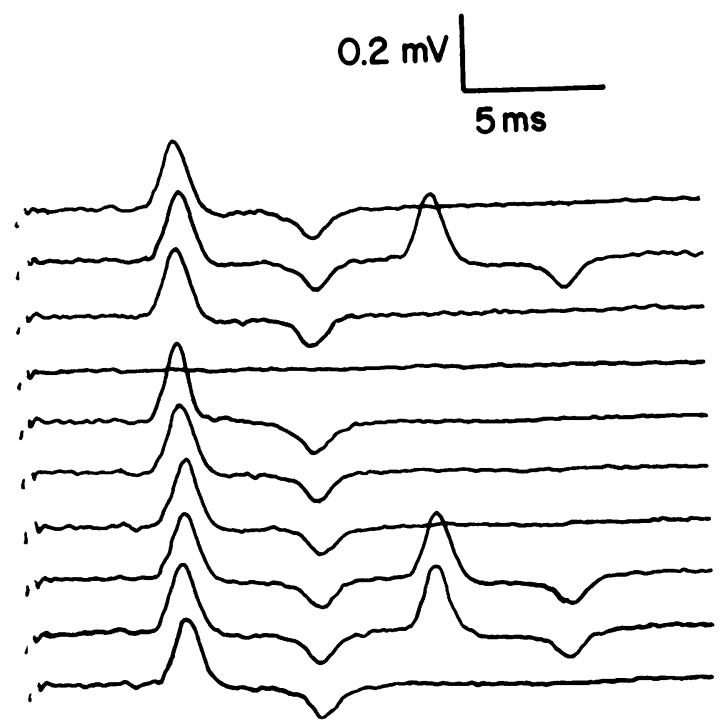

Fig. 3 Recording of single motor unit discharge using surface electrodes. Stimulation of a single motor fibre at stimulus intervals of $1.0 \mathrm{~s}$. The train begins at the bottom. Note the all or nothing character of the $M U$ discharge and the occurrence of the identical $M U$ in the $F$ recurrent discharge. $A$ sensory prepotential with a latency of $3.4 \mathrm{~ms}$. is just visible preceding the $M$ MU potential.
Milner-Brown, 1976); and (3) if an F recurrent discharge occurred, the size and configuration of the $\mathbf{F}$ unit was identical to the $\mathrm{M}$ unit.

Single MU latency (conduction velocity)

Measurement of MU terminal conduction times can be difficult, especially if the MU signal to noise ratio is low or the stimulus artefact hard to identify, or in situations where artefact contributions to the surface voltage are present. Such artefacts can be caused by antidromic sensory nerve action potentials or volume conduction and movement from excitation and contraction of proximal, usually forearm muscles, evoked by motor nerve stimulation at points close to or proximal to the elbow. For these reasons, only MUs in which the latency could be measured accurately were included.

In less than $5 \%$ of MUs, the identical MU could be excited at two or more points at least $20 \mathrm{~mm}$ apart. The conduction velocities of other MUs could not be measured directly. Therefore, the terminal conduction times of MUs excited at different distances from the motor point were adjusted to a standard distance $(200 \mathrm{~mm})$ from the motor unit point (Kadrie et al., 1976) using the following equation where $d$ is the distance from point of stimulation of the MU to motor point and $\mathrm{RL}=$ residual latency.

$$
\left(\begin{array}{l}
\text { Terminal motor } \\
\text { conduction time }
\end{array}-\mathrm{RL}\right) \times \frac{200}{\mathrm{~d}}+\mathrm{RL}
$$

The distance $200 \mathrm{~mm}$ was chosen because it was, for most subjects, the approximate midpoint of the lengths of the ulnar and median nerves open to surface stimulation. The inclusion of the $\mathrm{RL}$ in the calculation of adjusted latency is important because the $R L$ is a much larger fraction of the terminal motor conduction times for MUs excited near the wrist than those excited near the elbow or in the upper arm. The fact that the residual latencies for single motor fibres cannot be measured is a problem because it is difficult to know how representative the RL for the maximum compound potential is for all MUs. The residual latency is likely to be less for faster than slower conducting fibres. Therefore, because the residual latency was obtained from supramaximal stimulation, the approximations inherent in the adjusted latencies and calculations of single $\mathrm{MU}$ conduction velocities are likely to be most accurate for the fast conduction velocity fibres.

Calculation of the respective impulse velocities in single motor units was made by:

Conduction velocity (metres per second) $=$ 200 $\overline{\text { adjusted latency-RL }}$ 
NEUROMUSCULAR TRANSMISSION TESTS

The tests were carried out at room temperature $\left(24-26^{\circ} \mathrm{C}\right)$. Because of the large number of MUs to be tested, and limitations in the abilities of test subjects to maintain relaxation in the test muscles, the intervals between test trains were reduced from the period of two minutes suggested by Slomic et al. (1968) to 0.5 minute. Trains of 10 or more stimulus pulses at time intervals of 1.0 , 0.3 , and 0.1 second were used to test for changes in the parameters of single MUs and the MCP, the latter in response to supramaximal stimuli. Stimulus trains using interpulse intervals of more than one second were occasionally used if changes were obvious in the surface voltage at intervals of one second.

Tetanic stimulation at pulse intervals of 0.2 second for 30 seconds was used to test for posttetanic potentiation (Desmedt, 1973) in representative small, medium, and large MUs. At intervals of one minute, for 10 minutes after the tetanus, short test trains of stimulus intervals of 0.3 second were applied.

\section{Results}

MAXIMUM COMPOUND POTENTIAL (MCP)-

SUPRAMAXIMAL NERVE STIMULATION

Observations in the median thenar and ulnar hypothenar groups were nearly identical. Therefore, except in situations where a significant difference between observations in the ulnar and median groups occurred, observations reported below are specifically on the larger ulnar group. In Table 1 the residual latencies and the number of MUs for each of the 12 ulnar and two median nerves are listed.

In response to supramaximal trains of stimuli delivered at the level of the wrist, the $p-p V$ increased and the p-pV decreased, in an approximately proportional way (Figs. 4 and 5). The percentage change in $\mathrm{p}-\mathrm{pV}$ and $\mathrm{p}-\mathrm{pD}$ increased as the stimulus interval was reduced. The percentage change in $\mathrm{p}-\mathrm{pV}$ and $\mathrm{p}-\mathrm{pD}$ at any particular stimulus interval was usually maximum in the fifth compared with the first evoked maximum compound potentials in the train, significant changes being evident even at 1.0 second intervals. In certain cases it was evident that the changes in the MCP voltage lasted for as long as 10 minutes after a test train. For this reason, supramaximal stimulation was usually carried out at the end of the experiment to avoid producing long-term changes in MUs yet to be tested. This step was particularly important in pathological cases (Kadrie et al., 1976).
Table 1 Residual latencies and number of motor units for 12 ulnar and two median nerves

\begin{tabular}{|c|c|c|c|}
\hline Nerve & $\begin{array}{l}\text { Residual } \\
\text { latency } \\
(m s)\end{array}$ & $\begin{array}{l}\text { Maximum } \\
\text { conduction } \\
\text { velocity } \\
(400 \mathrm{~mm}) \\
(\mathrm{m} / \mathrm{s})^{*}\end{array}$ & $\begin{array}{l}\text { Motor } \\
\text { unit } \\
\text { number }\end{array}$ \\
\hline \multicolumn{4}{|c|}{ Hypothenar-ulnar } \\
\hline 1 & 2.5 & 60.1 & 18 \\
\hline 2 & 1.9 & 62.5 & 10 \\
\hline 3 & 1.8 & 65.6 & 10 \\
\hline 4 & 2.6 & 46.5 & 8 \\
\hline 5 & 2.3 & 50.6 & 4 \\
\hline 6 & 2.7 & 50.6 & 6 \\
\hline 7 & 2.1 & 61.5 & 7 \\
\hline 8 & 2.4 & 69.0 & 4 \\
\hline 9 & 2.6 & 62.5 & 4 \\
\hline 10 & 2.2 & 60.2 & 4 \\
\hline 11 & 2.1 & 70.1 & 6 \\
\hline \multirow[t]{2}{*}{12} & 2.2 & 62.5 & 5 \\
\hline & & & $\begin{array}{l}\mathrm{MU} \\
\text { total }=86\end{array}$ \\
\hline $\begin{array}{l}X \pm 1 S D \\
\text { Thenar-median }\end{array}$ & $2.3 \div 0.3$ & $60.1 \pm 7.4$ & \\
\hline 1 & 2.4 & 53.7 & 21 \\
\hline 2 & 3.1 & 62.5 & 18 \\
\hline
\end{tabular}

*See text

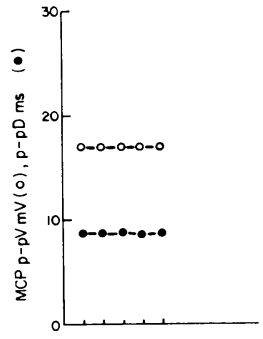

$10 \mathrm{~s}$

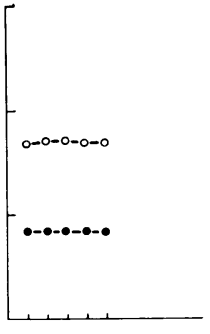

$03 \mathrm{~s}$

STIMULUS INTERVALS

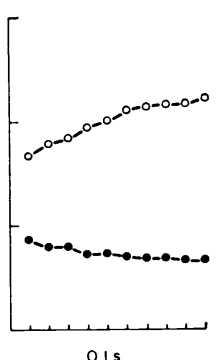

Fig. 4 Changes in the p-pV (in $m V$ ) and $p$-p duration (in $\mathrm{ms}$ ) of a control hypothenar maximum compound potential in response to supramaximal stimulation of the ulnar nerve at stimulus intervals of 1.0,0.3, and 0.1 second. Note increase in the $p-p V$ and reciprocal reduction in $p-p D$ at stimulus intervals of 0.1 second.

\section{SINGLE MOTOR UNITS}

Motor unit potentials have been classified into small (less than $0.05 \mathrm{mV} \mathrm{p}-\mathrm{p})$, medium (0.05$0.149 \mathrm{mV} \mathrm{p}-\mathrm{p})$, and large $(0.150 \mathrm{mV} \mathrm{p}-\mathrm{p})$. In the previous communication MUs were reported to be recruited in order from small to large by increments in the stimulus intensity (Kadrie et al., 1976). In this investigation, also, most MUs recruited first at points of stimulation were small, larger MUs being recruited second. The surface p-pV histogram for hypothenar MUs (total 58 MUs) from two control subjects is illustrated in Fig. 6, both the first and second MUs recruited; all stimulation points being included. In this in- 


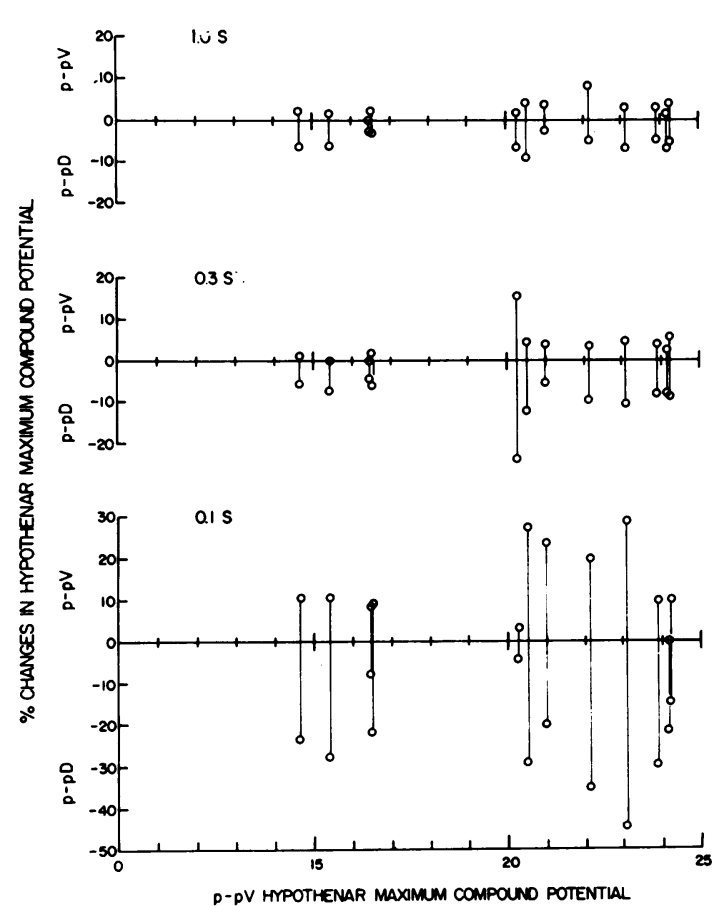

Fig. 5 Illustration of percentage changes in $p-p V$ and $p-p D$ at stimulus intervals of $1.0,0.3$, and 0.1 second for the maximum hypothenar compound potentials evoked by supramaximal ulnar nerve stimulation in 12 control subjects. The vertical bars connect corresponding $p-p V$ and $p-p D$ measurements for each nerve. Note that the percentage changes are maximum at stimulus intervals of 0.1 second in all but one of the control nerves.

vestigation, however, a deliberate attempt was made to include more of the medium and large MUs, and this is reflected in the histogram of MU sizes used for test trains of stimuli (total $64 \mathrm{MU}$ ) (Fig. 2). Persistent moving about of the stimulating electrode along the length of the nerve, frequently rejecting points where small MUs are obtained first, is rewarded by the isolation of more large and medium MUs as the first MU recruited; the range and distribution being more like that obtained by isometric contraction (Kadrie et al., 1976). In later communications the range and distribution of MU surface voltages in pathological states is to be compared with the range and distribution of MUs obtained by random selection of stimulation points and not the biased sampling method used in this particular investigation.

Motor units are recruited in order from small to large surface $\mathrm{p}-\mathrm{pV}$ and long to shorter latency by increments in the stimulus intensity. The first

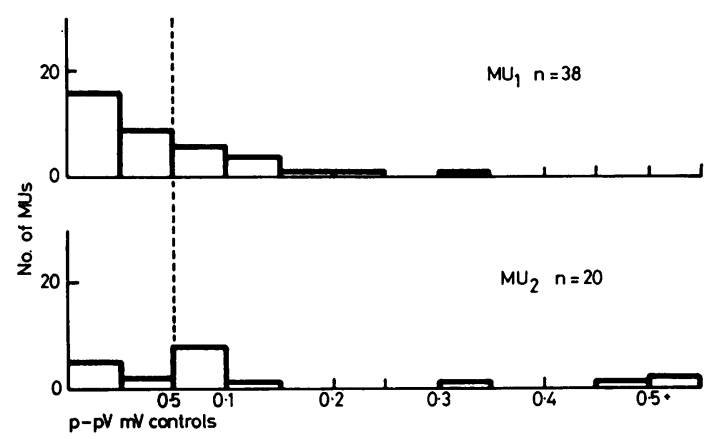

Fig. 6 Histogram of all motor units recruited first and second at all points of stimulation; there was no attempt to exclude small MUs or include more large MUs.

evidence for this was based on the finding of a significant correlation between the recruitment order of MUs at single points of stimulation and the respective MU surface $\mathrm{p}-\mathrm{pVs}$, areas, and latencies. The evidence was supported in this investigation by the highly significant correlation observed between, in this case, the surface p-pV and the latencies of MUs adjusted to $200 \mathrm{~mm}$, for each subject (Table 2). Plotting the latencies adjusted to a standard $200 \mathrm{~mm}$ from the motor point against the corresponding $\mathrm{MU}$ p-pV for all hypothenar MUs illustrates the fact that there is considerable overlap in the adjusted latencies of hypothenar MUs (Fig. 7). In general, however, the larger units tend to have short latencies and the longest latency MUs are usually small in p-pV. Perhaps more obviously meaningful is the conversion of the adjusted latencies (median and ulnar combined) to conduction velocities (Fig. 8). This histogram, constructed from the 78 hypothenar and thenar MUs in which the latency could be measured accurately, demonstrates the fact that only a minority of MUs actually determine the maximum motor conduction velocity and that there is a considerable range in the conduction velocities of individual MUs. The relationship between conduction velocity (or adjusted latency)

Table 2 Correlation between surface p-pv and latencies of motor units adjusted to $200 \mathrm{~mm}$

\begin{tabular}{llll}
\hline & \multicolumn{3}{l}{ Decreasing adjusted latency } \\
\cline { 2 - 4 } Total number of pairs 92 & + & - & ties \\
\hline Increasing p-pV & 67 & 23 & 2 \\
number of pairs & 73 & 25 & 2 \\
Percentage & & & \\
\hline
\end{tabular}

For statistical methods see Kendall (1962) and Kadrie et al. (1976). 


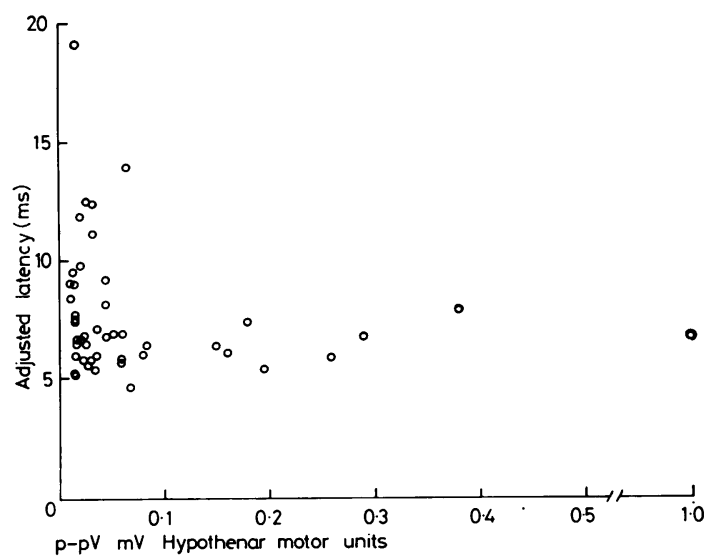

Fig. 7 Plot of the p-pV in $m V$ of hypothenar motor units against the corresponding latencies to be expected if the motor axons had all been excited at $200 \mathrm{~mm}$ from the motor point.

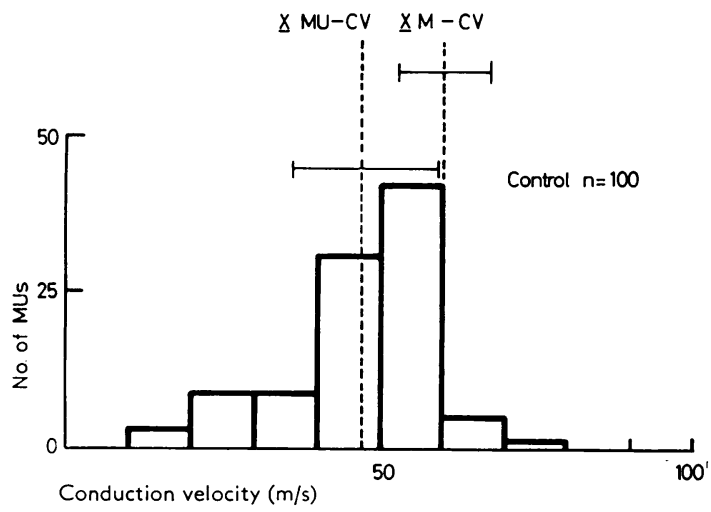

Fig. 8 Histogram of the conduction velocities of all hypothenar and thenar motor units for which the latencies could be measured accurately. The means $\pm 1 S D$ for the maximum motor conduction velocities (calculated for $400 \mathrm{~mm}$ from the motor point-see methods) and the MUs are designated by the interrupted lines and bars.

and surface voltage is important, because it illustrates that the surface $\mathrm{p}-\mathrm{pV}$ correlates closely to an important physiological parameter that itsell has been established to be related to the MU force (Milner-Brown et al., 1973; Freund et al., 1975; Milner-Brown and Stein, 1975). Therefore, in comparing the responses of single MUs to trains of stimuli it is not unreasonable to consider that the surface p-pV is a useful index of the probable general size small, medium, or large of the MU.
The stimulus intensities required to excite single MUs were usually half or less of the current required to stimulate the motor nerve at the same point just maximally. The stimulus currents required to excite single MUs were not usually uncomfortable even at high tetanic stimulus frequencies. In response to single MU stimulation movement was only occasionally observed in the corresponding hypothenar or thenar muscles. Therefore, movement artefact was probably negligible in this type of test. One minor drawback was that it was sometimes difficult to stimulate at shorter stimulus intervals $(0.1$ second or less) in the upper arm because of displacement of the stimulating electrode by contraction of underlying muscles evoked by direct spread of the stimulus current.

Single MU responses to repetitive stimulation paralleled the corresponding changes in the maximum compound potentials. Increases in the $p-p V$ linked to reductions in the p-pD of the surface MU potentials occurred at stimulus pulse intervals of $1.0,0.3$, and 0.1 second; the maximum percentage changes being observed at stimulus intervals of 0.1 second (Fig. 9). The maximum percentage changes at stimulus intervals of $1.0,0.3$, and 0.1 second were usually observed in the fifth $\mathrm{MU}$ potential in the train although occasionally the maximum changes were observed in the fourth or sixth potential. No significant differences in the percentage changes in $\mathrm{p}-\mathrm{pV}$ or $\mathrm{p}$-pD were observed comparing small, medium, and large MUs. The larger scatter noted in the observations in small MUs was probably the result of the much lower signal to noise ratios. In all hypothenar MUs, however, irrespective of size, the maximum decrement in $\mathrm{p}-\mathrm{pV}$ was less than $10 \%$. Therefore, in disease states to be reported in later communications, decrements in the p-pV of more than $10 \%$ have been considered abnormal.

The mechanism underlying the increments in $\mathrm{p}-\mathrm{pV}$ and corresponding reductions in the $\mathrm{p}-\mathrm{pD}$ was probably, in part, the result of a progressive reduction in the time intervals between MU component spikes (Fig. 10). In only three examples was it possible to record two or more distinct single fibre spikes that together fired in an all or nothing manner in response to threshold peripheral nerve stimulation. For most stimulus points the SFEMG electrode, located close to the endplate zone, at the start of the experiment, recorded an intramuscular MU potential corresponding to the surface MU potential, the two potentials always occurring together in an all or nothing manner. Only in a minority of MUs, however, could single muscle fibre spikes be obtained. In such single 


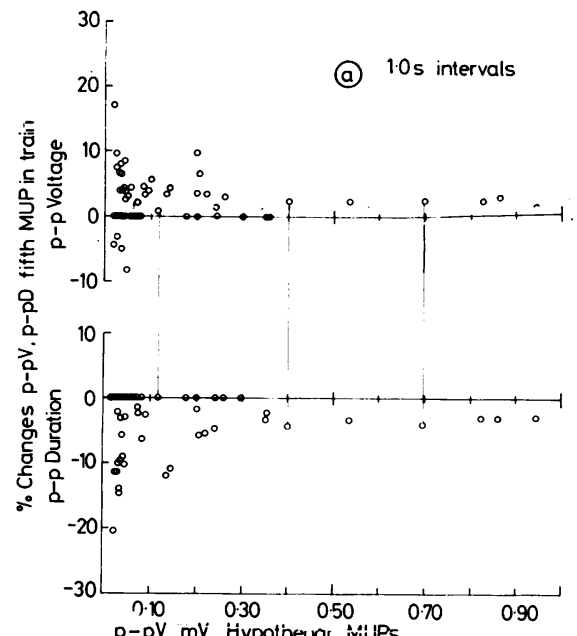

H. A. Kadrie and W. F. Brown

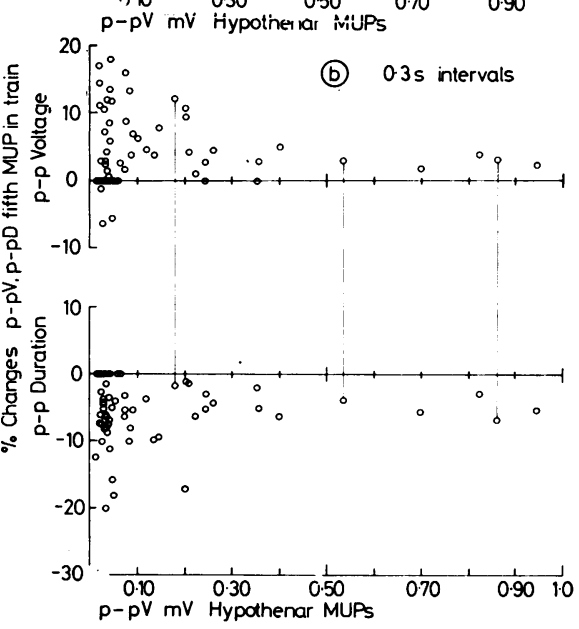

fibre spikes, an invariable progressive reduction in latency from stimulus to spike was observed in response to trains of stimuli, the reduction being maximum at the shorter stimulus intervals. In the three examples in which two spikes could be obtained the progressive reduction in latency, usually maximum at the time of the fifth MU potential in the train, was invariably more in the longer latency spike compared to the first spike. The result was a progressive reduction of the interspike interval (Fig. 10). This increase in the synchronisation in firing times of MU component muscle fibres could account for the increase in $\mathrm{p}-\mathrm{pV}$ and reduction in $\mathrm{p}-\mathrm{pD}$ observed in single MUs and in the maximum compound potentials evoked by trains of stimuli and to which the term pseudofacilitation has been applied by Desmedt (1973). The number of single and double spike recordings could have been increased by adjustment of the SF-EMG electrode but this proved time-consuming and uncomfortable to the test subjects and was, therefore, abandoned.

Tetanic stimulation at stimulus intervals of 0.02 second evoked still larger increases in the p-pV and reductions in the $\mathrm{p}-\mathrm{pD}$ compared to intervals of 0.1 second. After the tetanus, especially in the larger MUs, increases in MU p-pV lasting for as long as 10 minutes (Fig. 11) were observed, probably also the result of increased synchronisation of MU muscle fibre action potentials (Desmedt, 1973).

The relation between surface $\mathrm{p}-\mathrm{pV}$ and the corresponding adjusted latency (or, conversely, conduction velocity) reported before for hypothenar MUs (Fig. 7) did not hold in thenar-median MUs (Fig. 12). No significant differences were noted in the range and distribution of the surface $p-p V$ of the thenar MUs compared to hypothenar MUs but in thenar MUs no systematic relationship of MU p-pV to adjusted latency was obvious. The range in adjusted latencies or MU conduction velocities was not as wide in the two median nerves tested as in the four ulnar nerve in which the measurements and calculations were made.

Fig. 9 Illustration of the percentage changes in $p-p V$ and $p-p D$ of the fifth compared to the first motor unit potential evoked by stimulus pulses at intervals of (a) $1.0,(b) 0.3$, and (c) 0.1 second. These changes are plotted against the corresponding $p$-p voltage in $m V$ for all the corresponding $M U$ on the $X$ axis. The vertical bars illustrate the reciprocal nature of the percentage changes in $p-p V$ and $p-p D$ for three $M U s$. Note (1) no more than a $10 \%$ decrement $p-p V$ was observed for any single $M U$, and (2) the larger range in percentage change of $p-p D$ and $p-p V$ observed in $M U<0.2 m V p-p V$. 

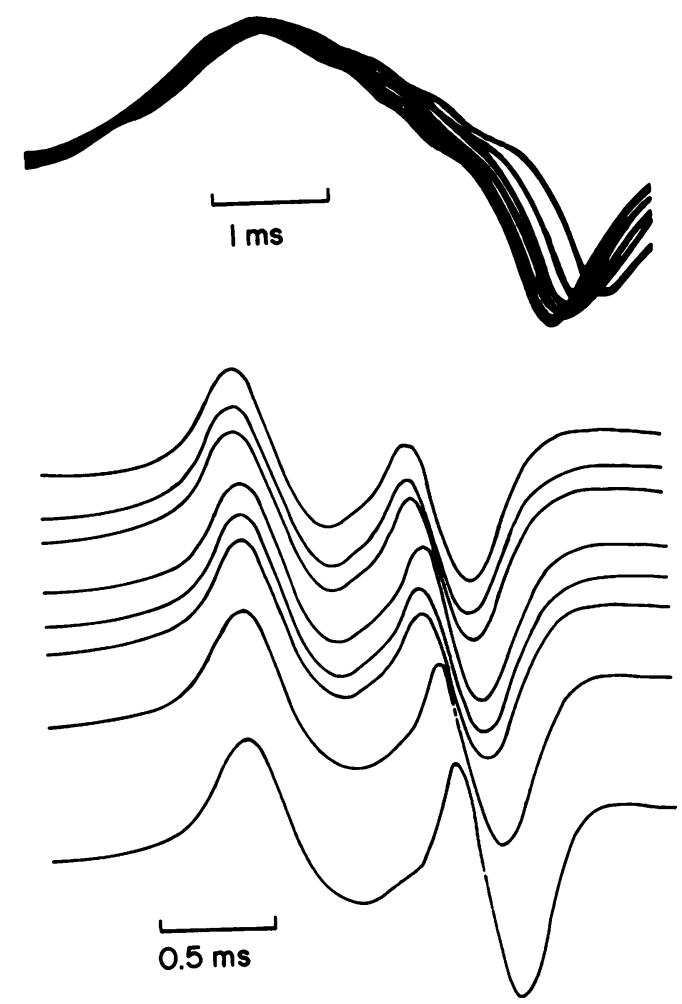

Fig. 10 Recording of the surface MU potential (upper) and the corresponding intramuscular recording (lower) of two single fibre spikes. Successive traces evoked by a stimulus train at 0.1 second intervals are superimposed in the upper surface motor unit potential recording and in raster display, the two intramuscular spikes in the lower illustration. The train sequence is from bottom to top and illustrates a progressive reduction in the interspike interval of $0.19 \mathrm{~ms}$. In the surface motor unit potential, a progressive increase in the p-pV and reduction in the p-pD occurred. Both recordings are delayed for display on an expanded horizontal sweep.

The reasons for this lack of correlation between conduction velocity and surface voltage in thenar MUs is not known.

\section{Discussion}

From experience in this investigation it is obvious that neuromuscular transmission can be successively tested in five or more individual MUs from thenar or hypothenar muscles in man. The technique has the advantage that stimulation, even at high frequencies, is usually not uncomfortable to patients because of the lower stimulus currents required, unlike supramaximal nerve stimulation.

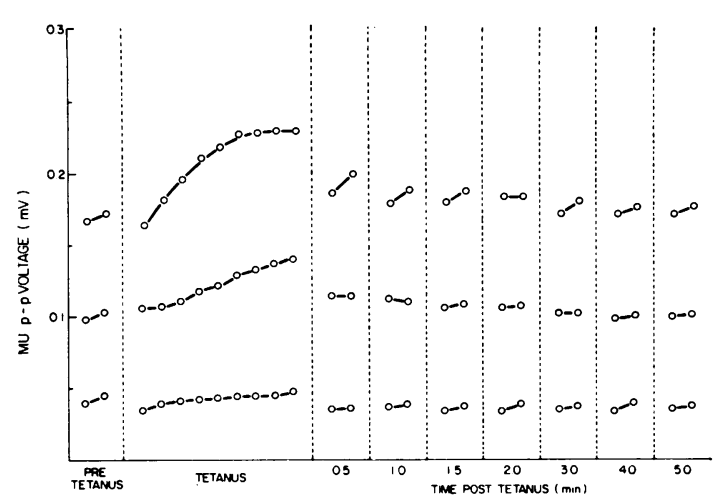

Fig. 11 Changes in p-p voltage of the fifth compared to the first motor unit potential evoked by stimuli at 0.3 second intervals, pretetanus, and at various times after tetanus. The tetanus was delivered at 0.02 second intervals. For the tetanus, only every third potential in the train is plotted. Three representative $M U$ responses are plotted. The increases in p-p voltage in the tetanus were paralleled by reciprocal changes in the p-p duration. Note particularly in the largest of the three MUs that, for up to two minutes after tetanus, the first and fifth motor unit potentials evoked were larger than the corresponding pretetanus values.

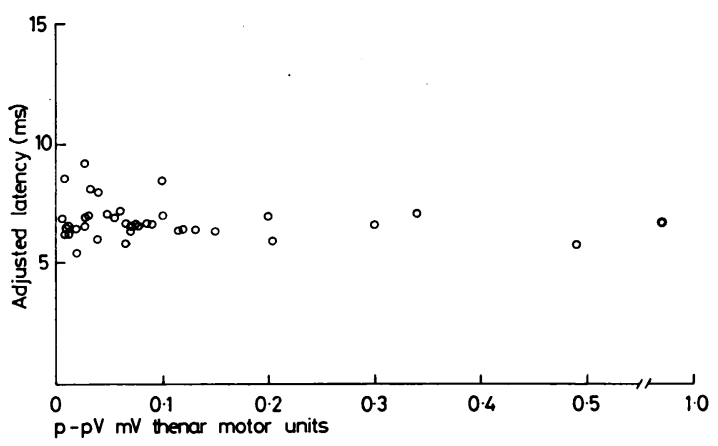

Fig. 12 Plot of the p-pV in $m V$ of thenar motor units against the corresponding latencies to be expected if the motor axons had all been excited at $200 \mathrm{~mm}$ from the motor point.

Moreover, there is no significant contribution from movement artefact, a major problem using supramaximal stimulation (Slomic et al., 1968; Ozdemir and Young, 1971, 1976; Desmedt and Borenstein, 1976). From a physiological viewpoint, the method is of value and interest because it tests the functional unit of the motor system, the motor unit. The method, as a consequence, could be potentially much more sensitive if abnormalities in neuromuscular transmission were present in a limited proportion of MUs only, perhaps selec- 
tively involving specific functional types of motor units. Abnormalities in MUs would not, therefore, be obscured by inclusion of more normal MUs as in the case of supramaximal stimulation.

The method also makes it possible to combine a look at the overall action of the MU that is reflected in the surface summation voltage and at the component single neuromuscular junctions by recording in parallel from the surface and single fibre EMG electrodes. The latter permits measurement of the variations in firing time of single muscle fibre spikes and the recognition in pathological states of the failure of spike generation at single junctions (Stalberg and Ekstedt, 1973; Stalberg et al., 1974; Schwartz and Stalberg, 1975). In this sense, the surface voltage provides an index of the approximate total size of the unit (Milner-Brown and Stein, 1975; Kadrie et al., 1976) representing the summation of the voltages from all the muscle fibres in the MU, factors such as the particular geographic position of the MU with respect to the surface electrode and the degree of synchronisation of muscle fibre action potentials being incorporated into that sum. The single fibre electrode provides an index in much more detail of one to three individual junctions in the MU. This combination of intramuscular and surface recording has proved to be helpful in pathological states.

Disadvantages of testing neuromuscular transmission in single MUs are that the methods are not easy, requiring considerable experience to hold the stimulating electrode properly to maintain an MU in isolation for periods as long as 10 to 30 minutes. The low signal to noise ratios in small MUs moreover, make accurate measurements of changes in the p-pV, p-pD, and latency more difficult. In controls this latter problem can be partially compensated for by signal averaging. Averaging methods, however, increase the time required to investigate a motor unit, thus limiting the total number of MUs obtained per subject. Averaging, moreover, cannot be used in pathological states where the responses to successive trains of stimuli frequently differ. Despite the technical problems in all control subjects and pathological cases investigated to date, it has been possible to isolate and investigate at least four single units, and frequently more than 10 per subject. Desmedt and Borenstein (1970) had reported that only one MU can be isolated per nerve and moreover, that even one MU cannot be obtained in some subjects.

The changes in the maximum compound potential p-pV and p-pD observed in hypothenar and thenar muscles are in approximate agreement with earlier investigations (Slomic et al., 1968;
Desmedt and Borenstein, 1970; Desmedt, 1973). In the investigation by Slomic et al., the percentage increases in $\mathrm{p}-\mathrm{pV}$ at pulse intervals of 1 to 0.1 second were similar to those observed in this investigation. Decrements of more than $7 \%$ in the negative peak voltage when stimulated at 3 per second have not been observed in controls. (Desmedt and Borenstein, 1970). All previous investigations using supramaximal stimulation are in fundamental agreement with the present observations in that maximum changes in $\mathrm{p}-\mathrm{pV}$ or negative peak voltage and corresponding maximum reductions in p-pD occur at stimulus intervals of 0.1 , more than 0.3 , more than 1.0 second.

No comparable observations to this communication on large numbers of control single MUs have been reported. In general the changes in MU p-pV and $\mathrm{p}-\mathrm{pD}$ paralleled the changes in the maximum compound potentials. There were no significant differences in the changes in small, medium, and large MUs. The larger variations in the percentage changes of the small MUs were probably the result of larger measurement errors inherent in such low signal to noise MUs.

In the hypothenar-ulnar group of MUs there was an approximate relationship between the voltage of the MUs recorded on the skin surface and the latency adjusted to $200 \mathrm{~mm}$ or the corresponding conduction velocity. There was clearly a lot of overlap but, nonetheless, the larger MUs in general had short latencies and correspondingly faster conduction velocities, the longest latency and slowest conduction velocity MUs invariably having a lower surface $\mathrm{p}-\mathrm{pV}$. This relationship did not hold in the thenar median nerve group. In the latter, even though the ranges in latencies and surface $p-p V$ of thenar MUs were comparable to the corresponding hypothenar-ulnar nerve ranges, there was no significant coupling of latency or velocity to surface voltage. This observation was not expected. It is worth pointing out in this regard that the relationships between tensions and conduction velocity (Freund et al., 1975) and between surface voltage and force (Milner-Brown and Stein, 1975) were established for the ulnar innervated first dorsal interosseous and hypothenar muscles (Kadrie et al., 1976) even though in the last investigations at single points of stimulation there was a significant ranking of thenar MU surface voltage and latency to order of recruitment.

The lack of correlation between adjusted latency (or conduction velocity) and surface voltage in the thenar muscle group must make us, therefore, even more cautious about using the surface $p-p V$ as an index to the probable functional size of thenar MU. For this reason, most of the patho- 
logical investigations have been concentrated in a hypothenar-ulnar nerve group. Even in those cases, however, surface $\mathrm{p}-\mathrm{pV}$ as a guide to probable $\mathrm{MU}$ size must be used cautiously. In our view, it is probably valid, however, to classify MUs into three surface voltage ranges, small, medium, and large; ranges that probably can be accepted as approximate guides to a probable MU size. Unfortunately, it was not possible to measure the force generated by electrically excited single motor axons because of the complex factors involved.

The calculation of the adjusted latencies and the corresponding conduction velocities must be included because these values provide indices in addition to the surface voltage to characterise the probable size and functional type of MU. There are a series of potential technical and theoretical errors inherent in such calculations. The first, of course, is the error in measurement of the terminal latency itself. The measures taken to recognise and limit this error have been discussed in the methods. A second, theoretical, error is that the residual latency obtained using supramaximal stimulation at three or more points along the length of the nerve probably best reflects the residual latency in the faster conducting fibreslower conduction velocity fibres are likely to have correspondingly longer residual latencies.

Unfortunately, there is no way to measure the residual latencies of single fibres in man because the measurement requires stimulation of the identical fibre at two or more points along the length of the nerve, an occurrence that did not happen once in the control median and ulnar nerves although occurring more often in pathological nerves. The residual latency correction is particularly important for fibres stimulated close to the motor point because the residual latency represents such a large fraction of the terminal latency. Errors, therefore, in the adjusted latency are likely to be the largest for fibres excited close to the motor point and for slower conduction velocity fibres excited at any level.

Whatever the error in these calculations, it is interesting how much agreement there is in the range in conduction velocities over all the controls. The maximum conduction velocity obtained by supramaximal stimulation clearly corresponds to the conduction velocities of the faster conducting fibres although an occasional few fibres have faster calculated velocities. The range in MU conduction velocities is wider than expected from other methods (Gilliatt et al., 1976). This method is useful, however, because it provides another index to the probable MU size.

The investigation has established that "pseudo- facilitation" is observed not only in the maximum compound potential in response to supramaximal stimulation, but in component single MUs of all sizes. Pseudofacilitation is likely to be the result of better synchronisation in the summation of single muscle fibre spikes in the MUs (Desmedt, 1973) but the exact mechanism cannot be considered established. Repetitive stimulation of single muscle fibres has been observed to increase their conduction velocity (Stalberg, 1966) but that factor, if equally distributed in all muscle fibres, would not necessarily result in more synchronous summation of muscle fibre spikes. Such an increase in the degree of synchronisation must depend on interaction between muscle fibres, most likely between fibres that are immediate membrane neighbours in the muscle in order to permit a significant change in the excitability of muscle fibre membranes to be changed by nearby propagated action potentials of muscle fibres as in the case of nerve fibres (Katz and Schmidt, 1940).

Only a low proportion of muscle fibres in healthy single MUs, however, are placed back to back (Brandstater and Lambert, 1973; Kugelberg, 1973). Theoretically, therefore, synchronisation of muscle fibres spikes could only occur in that fraction of muscle fibres in the MU that are grouped in pairs or triplets but not in isolated single fibres. Synchronisation could be expected to be higher in situations where the proportion of muscle fibres grouped together is higher, as in certain peripheral neuropathies, a point to be discussed in more detail in later communications using this method.

For the present, it has simply been observed in this investigation that in situations where two single fibre spikes could be clearly recorded, in an all or nothing manner corresponding to the response of a single MU, repetitive stimulation, particularly at stimulus intervals of 0.3 and 0.1 second resulted in significant reductions in the interspike interval corresponding to an increase in the p-pV and reduction in the p-pD of the surface voltage of the MU. This observation is in accordance with that of Schwartz and Stalberg (1975) who also observed a progressive reduction in the latency of MU spikes in response to the first few stimuli of a train although they did not comment specifically on the interspike interval which is, after all, the critical parameter reflecting the degree of synchronisation in firing of MU muscle fibre potentials.

We wish to thank the subjects who served as controls, Mrs T. Stewart for typing the manuscript, Mr G. Moogk for the illustrations, and $\mathrm{Mr}$ S. Yates for technical help. 


\section{References}

Bergmans, J. (1970). The Physiology of Single Human Nerve Fibres. Vander: Brussels.

Borenstein, S., and Desmedt, J. E. (1973). New diagnostic procedures in myasthenia gravis. In New Developments in Electromyography and Clinical Neurophysiology. Vol. 1, pp. 350-374. Edited by J. E. Desmedt. Karger: Basel.

Brandstater, M. E., and Lambert, E. H. (1973). Motor unit anatomy. Type and spatial arrangement of muscle fibres. In New Developments in Electromyography and Clinical Neurophysiology. Vol. 1, pp. 14-22. Edited by J. E. Desmedt. Karger: Basel.

Brown, W. F., and Milner-Brown, H. S. (1976). Some electrical properties of motor units and their effects on the methods of estimating motor numbers. Journal of Neurology, Neurosurgery, and Psychiatry, 39, 249-257.

Desmedt, J. E. (1973). The neuromuscular disorder in myasthenia gravis. I. Electrical and mechanical response to nerve stimulation in hand muscles. II. Pre-synaptic cholinergic metabolism, myastheniclike syndromes and a hypothesis. In New Developments in Electromyography and Clinical Neurophysiology. Vol. 1, pp. 241-342. Edited by J. E. Desmedt. Karger: Basel.

Desmedt, J. E., and Borenstein, S. (1970). The testing of neuromuscular transmission. In Handbook of Neurology, Vol. 7, pp. 104-115. Edited by P. J. Vinken and G. W. Bruyn. North Holland: Amsterdam.

Desmedt, J. E., and Borenstein, S. (1976). Diagnosis of myasthenia gravis by nerve stimulation. Annals of the New York Academy of Sciences, 274, 174188.

Freund, H. J., Budingen, H. J., and Dietz, V. (1975). Activity of single motor units from human forearm muscles during voluntary isometric contractions. Journal of Neurophysiology, 38, 933-946.

Gilliatt, R. W., Hopf, H. C., Rudge, P. ,and Baraitser, M. (1976). Axonal velocities of motor units in the hand and foot muscles of the baboon. Journal of the Neurological Sciences, 29, 249-258.

Kadrie, H. A., Yates, S. K., Milner-Brown, H. S., and Brown, W. F. (1976). Multiple point electrical stimulation of ulnar and median nerves. Journal of Neurology, Neurosurgery, and Psychiatry, 39, 937985.

Katz, B., and Schmidt, O. H. (1940). Electric interaction between two adjacent nerve fibres. Journal of Physiology, 97, 471-488.

Kendall, M. G. (1962). Rank Correlation Methods. Hofner: New York.
Krarup, C. (1977a). Electrical and mechanical responses in the platysma and in the adductor pollicis muscle: in normal subjects. Journal of Neurology, Neurosurgery, and Psychiatry, 40, 234-240.

Krarup, C. (1977b). Electrical and mechanical responses in the platysma and in the adductor pollicis muscle: in patients with myasthenia gravis. Journal of Neurology, Neurosurgery, and Psychiatry, 40, 241-249.

Kugelberg, E. (1973). Properties of the rat hind-limb motor units. In New Developments in Electromyography and Clinical Neurophysiology. Vol 1, pp. 213. Edited by J. E. Desmedt. Karger: Basel.

Milner-Brown, H. S., and Stein, R. B. (1975). The relation between the surface electromyogram and muscular force. Journal of Physiology, 246, 549-569.

Milner-Brown, H. S., Stein, R. B., and Yemm R. (1973). The contractile properties of human motor units during voluntary isometric contractions. Journal of Physiology, 228, 285-306.

Ozdemir, C., and Young, R. R. (1971). Electrical testing in myasthenia gravis. Annals of the New York Academy of Sciences, 183, 287-302.

Ozdemir, C., and Young, R. R. (1976). The results to be expected from electrical testing in the diagnosis of myasthenia gravis. Annals of the New York Academy of Sciences, 274, 203-222.

Schwartz, M. S., and Stalberg, E. (1975). Single fibre electromyographic studies in myasthenia gravis with repetitive nerve stimulation. Journal of Neurology, Neurosurgery, and Psychiatry, 38, 678-682.

Simpson, J. A. (1974). Myasthenia gravis and myasthenic syndromes. In Disorders of Voluntary Muscle, 3rd Edition, pp. 653-692. Edited by J. N. Walton. Churchill Livingstone: Edinburgh and London.

Electrical and mechanical responses of normal and

Slomice, A., Rosenfalck, A., and Buchthal, F. (1968). myasthenic muscle, with particular reference to the staircase phenomenon. Brain Research, 10, 1-78 (special issue).

Stalberg, E. (1966). Propagation velocity in human muscle fibres. Acta Physiologica Scandinavica, 70, supplement 287, 1-112.

Stalberg, E., and Ekstedt, J. (1973). Single fibre EMG and microphysiology of the motor unit in normal and diseased human muscle. In New Developments in Electromyography and Clinical Neurophysiology, Vol. 1, pp. 113-129. Edited by J. E. Desmedt. Karger: Basel.

Stalberg, E., Ekstedt, J., and Broman, A. (1974). Neuromuscular transmission in myasthenia gravis studied with single fibre electromyography. Journal of Neurology, Neurosurgery, and Psychiatry, 37, 540-547. 